\title{
In Vitro Antimicrobial Activities of 6-Substituted-3(2H)- pyridazinone-2-acetyl-2- (substituted/nonsubstitutedbenzal/ acetophenone) Hydrazone Derivatives
}

\author{
Gul Bayram Abiha*, Leyla Bahar, Semra Utku \\ Mersin University, Mersin, Turkey
}

\begin{abstract}
Aim: In vitro antibacterial activity of 6-substituted-3(2H)-pyridazinone-2-acetyl-2-(substituted/nonsubstitutedbenzal/acetophenone) hydrazone derivatives were tested in common species causing hospital-acquired infections.

Material and Method: Antimicrobial activities of the compounds were performed by determining minimum inhibitory concentration (MIC) value against four Gram-positive, five Gram-negative and four Candida species fungi. Modified serial microdilution method was carried out. Reference strains of American Type Culture Collection (ATCC) were used.

Results: In general, eleven compounds exhibited considerable activity. Comparatively, compound 3 exhibited strong activity against Enterobacter hormaechei and 5, 11 were the most active against Acinetobacter baumannii at $31.25 \mu \mathrm{g} / \mathrm{mL}$. Compounds 1,2,3,4,8 and 10 were found to be as active as positive control ampicillin trihidrate against Stenotrophomonas maltophilia. On the other hand, compounds 1,2,3,4,7,8,9,10 and 11 showed strong antifungal activitiy as much as fluconazole against Candida tropicalis. Compound 1 was mostly active against Candida albicans, Candida glabrata, Candida parapsilosis and Candida tropicalis. It was also revealed that the antifungal activity of compounds 1, 6, 7, 8 and 9 were higher than the others. Compound 1 and 8 exhibited the best activity against Candida glabrata and Candida parapsilosis respectively.

Conclusions: All tested compounds showed better activity against Gram-negative bacteria and yeast than Gram-positive bacteria. These compounds may be considered as alternative antimicrobial agents in the treatment of multiple drug resistant Gram-negative, Gram-positive bacteria and fungal pathogens. Especially, we suggested that Compound 1 and 8 might be a promising candidate of new antifungal agents.
\end{abstract}

Keywords: antimicrobial, chemotherapeutic, microdilution, 3(2H)-pyridazinone

Received: $26^{\text {th }}$ October 2017; Accepted: $8^{\text {th }}$ April 2018; Published: $15^{\text {th }}$ April 2018

\section{Introduction}

Antimicrobial drugs still maintain their place as indispensable in combating infectious diseases in the world. However, increasing resis- tance to these drugs in recent years has reached a point of concern in the treatment of infectious diseases, especially in immunocompromised patients. Nowadays, there are various antibiotic

*Corresponding author: Gul Bayram Abiha, Mersin University, Mersin, Turkey

E-mail: gulbayram@mersin.edu.tr 
resistance mechanisms and multidrug-resistant microorganisms (1). Multidrug antimicrobial resistance among clinical strains is increasing at an alarming rate to public health. There is a strong need for new antimicrobials against Gram-negative, Gram-positive bacterial and fungal pathogens, causing community acquired and nosocomial infections (2). Today, there are many researches on various chemotherapeutic compounds to develop new antimicrobials $(3,4)$. Pyridazinone and its derivatives are one of these compounds commonly tested to overcome antimicrobial resistance (5). Recent researches have revealed that substituted pyridazinones have various chemical and biological activities such as anti-inflammatory and analgesic, antidepressant, anti-hypertensive, anticonvulsant, cardiotonic, antibacterial, antifungal, antitubercular, diuretics, anti-HIV, antimalarial and anti-cancer effects (6-9). To this effect, 3(2H)-pyridazinones first prepared by Fischer have a unique structure and display variety of pharmacological and therapeutic properties (10-13). It was reported that this special skeleton could be functional at various ring positions. Therefore, this structure attracts many scientists to synthesize and design new drugs. There are various studies reporting antibacterial, antitubercular and anti-fungal effects of these compounds (11-13). Anti-fungal activity of pyridazinone derivatives was detected against Candida albicans and Cryptococcus neoformans which cause secondary infections in immunosuppressive patients $(14,15)$.

Aim of this study is to investigate antibacterial (Bacillus subtilis subsp. subtilis, Enterococcus faecium, Staphylococcus aureus, Enterobacter hormaechei, Klebsiella pneumoniae, Acinetobacter baumannii, Pseudomonas aeruginosa, Escherichia coli O157H7, Stenotrophomonas maltophilia) and anti-fungal (Candida albicans, Candida glabrata, Candida parapsilosis, Candida tropicalis) activity of 6-substituted-3(2H)-pyridazinone-2-acetyl-2-(substituted/ nonsubstitutedbenzal/acetophenone) hydrazone derivatives by modified microdilution method. These hydrazone derivatives were synthesized and tested for their analgesic and anti-inflammatory or antimycobacterial activities previously by us $(16,17)$.

\section{Material and Method}

\section{Antimicrobial Activity Test}

Reference strains of American Type Culture Collection (ATCC, USA) were used. Reference microbial strains were B. subtilis subsp. subtilis (ATCC 6051), E. faecium (VanR QC set ATCC 700221), S. aureus (ATCC 29213), E. hormaechei (ATCC 700323), K. pneumoniae (ATCC 27736), A. baumannii (ATCC 49139), P. aeruginosa (ATCC 27853), E. coli O157:H7 (ATCC 35150), S. maltophilia (ATCC 17666), C. albicans (ATCC 14053), C. glabrata (ATCC 15126), C. parapsilosis (ATCC 22019), C. tropicalis (ATCC 1969). While lyophilized bacterial strains were inoculated to Mueller-Hinton agar (MHA) at $37^{\circ} \mathrm{C}$ for $24 \mathrm{~h}$, fungal strains inoculated to sabouraud dextrose agar (SDA) at $28^{\circ} \mathrm{C}$ for $24 \mathrm{~h}$. Microdilution method was applied by modification of the literature methods (18-20). Experiments were run in duplicate independently according to literature. Stock solutions of each compound were diluted in $\mathrm{DMSO} / \mathrm{H}_{2} \mathrm{O}(50 \%)$ at $1000 \mu \mathrm{g} / \mathrm{mL}$. The suspension of the bacteria and fungi strains were prepared in normal saline and the turbidity adjusted to $0.5 \mathrm{McF}$ arland with absorbance of $0.08-0.13$ at $625 \mathrm{~nm}$. For antibacterial activity test, $100 \mathrm{~mL}$ Mueller-Hinton Broth (MHB) was added to each of the 11 wells (in a 96-well plate). A $100 \mathrm{~mL}$ of tested compound solution was added to the first well, and two-fold dilutions were performed. Then, $10 \mathrm{~mL}$ bacterial suspension $\left(1 \times 10^{6} \mathrm{CFU} / \mathrm{mL}\right)$ was added to each well, except the last control well. Ampicillin trihydrate was used as reference drug. Plates were incubated at $37^{\circ} \mathrm{C}$ for $24 \mathrm{~h}$. 
For antifungal activity test, $100 \mathrm{~mL}$ Tryptic Soy broth (TSB) was added to each of the 11 wells. A $100 \mathrm{~mL}$ of tested compound solution was added to the first well, and two fold dilutions were performed. Then, $1 \mathrm{~mL}$ fungal suspension was added to each well, except the last one, acting as control well. Fluconazole was used as reference drug. Plates were incubated at $28^{\circ} \mathrm{C}$ for $24 \mathrm{~h}$. After incubation of all plates, the MICs were evaluated by spectrophotometer. The concentration resulting in a $50 \%$ reduction in the optical density (OD) values was compared to a reproduction control at $450 \mathrm{~nm}$ by spectrophotometric evaluation and defined as the MIC value.

In this study, descriptive analysis statistical method was used to evaluate the antibacterial activity of compounds. IBM SPSS free trial version was used as statistical package software.
Clinical isolates weren't tested in this study. Therefore, ethical approval was not required.

\section{Synthesis of Pyridazinone Compounds}

General procedure for synthesis of 6-substituted-3(2H)-pyridazinone-2-acetyl-2-(substituted-/ nonsubstitutedacetophenone) hydrazone derivatives 1-11 was shown in Scheme 1. Reaction of 3,6-dichloropyridazine with arylpiperazines afforded 3-chloro-6-substitutedpyridazine derivatives. Hydrolysis of 3-chloro-6-substitutedpyridazines were carried out upon heating in glacial acetic acid to afford 6-substituted-3 $(2 H)$-pyridazinone derivatives. The formation of these compounds were confirmed by IR spectra of a $\mathrm{C}=\mathrm{O}$ signal at about $1660 \mathrm{~cm}^{-1}$. Ethyl 6-substituted-3(2H)-pyridazinone-2-ylacetate derivatives were obtained by the reaction of 6-substituted-3(2H)-pyridazinone deriva-<smiles>[R]N1CCNCC1</smiles><smiles>[CH]1CCC1</smiles><smiles>[R]N1CCN(c2ccc(Cl)nn2)CC1</smiles><smiles>[R]N1CCN(c2ccc(=O)n(CC(=O)OCC)n2)CC1</smiles>

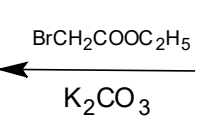<smiles>[Y]C(C)(C)C(=O)O</smiles>

$$
\downarrow \mathrm{NH}_{2} \mathrm{NH}_{2} \cdot \mathrm{H}_{2} \mathrm{O}
$$<smiles>[R]N1CCN(c2ccc(=O)n(CC(N)=O)n2)CC1</smiles>
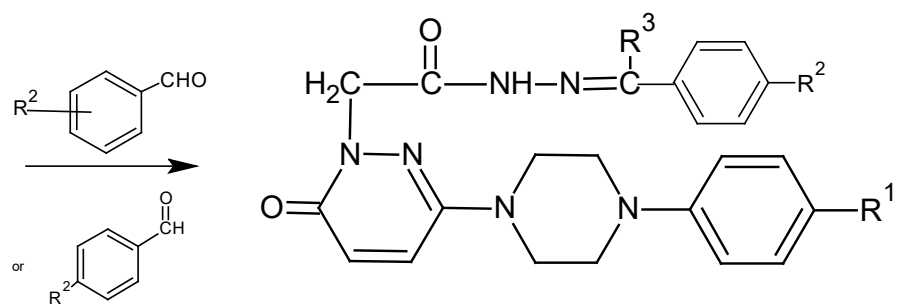

Compound 1-11

Scheme 1. Synthesis of compounds 1-11. 
tives with ethyl bromoacetate in the presence of $\mathrm{K}_{2} \mathrm{CO}_{3}$ in acetone. 6-Substituted-3(2H)-pyridazinone-2-yl-acetohydrazide derivatives were synthesized by the condensation reaction of ethyl 6-substituted-3(2H)-pyridazinone-2-ylacetate derivatives with hydrazine hydrate. A mixture of 6-substituted-3(2H)-pyridazinone-2-yl-acetohydrazide derivatives and appropriately substituted p-substituted acetophenone or benzaldehydes was refluxed in $15 \mathrm{~mL}$ of ethanol for 6 $\mathrm{h}$. The mixture was then poured into ice water. The formed precipitate was recrystallized from ethanol. Chemical structures of compound 1-11 are given in Table 1. 6-substituted-3(2H)-pyridazinone-2-yl-acetohydrazide derivatives 1-11 were synthesized as described previously by us as stated above (16-17). Elemental analysis, IR and ${ }^{1} \mathrm{H}-\mathrm{NMR}$ spectral data of the 1-11 were in accordance with the data reported previously (Table 2).

\section{Results}

\section{Antimicrobial Activity Assay}

The antimicrobial activities of target com-

Table 1. Chemical structures of the 6-Substituted-3(2H)-pyridazinone-2-acetyl-2-(substituted/ nonsubstitutedbenzal/acetophenone) hydrazone derivatives

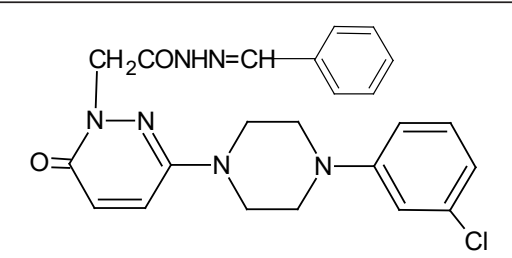

Compound 1

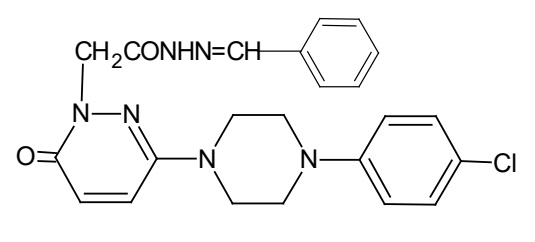

Compound 2

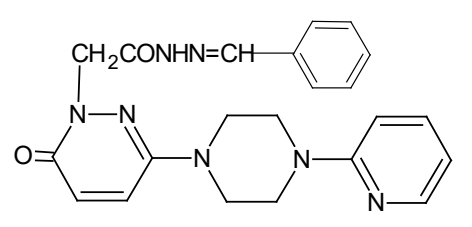

Compound 3

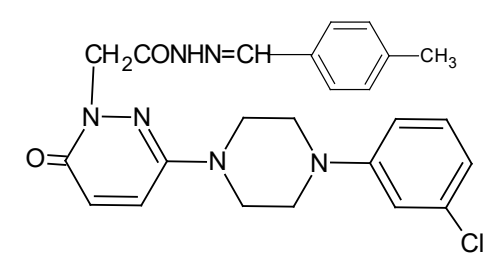

Compound 4

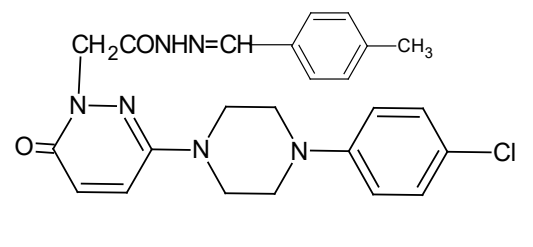

Compound 5

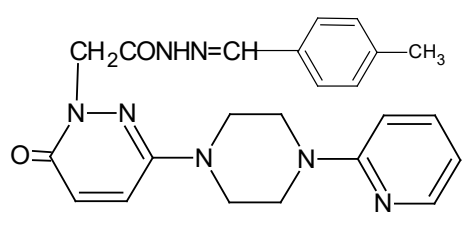

Compound 6
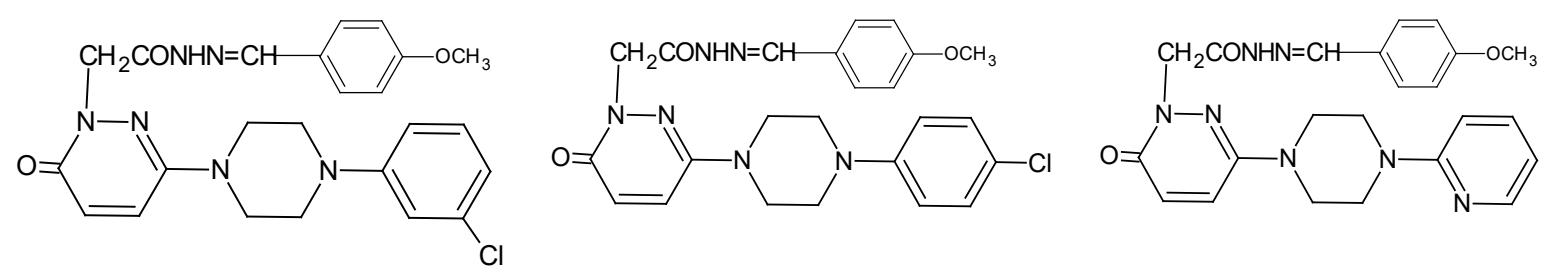

Compound 7

Compound 8

Compound 9
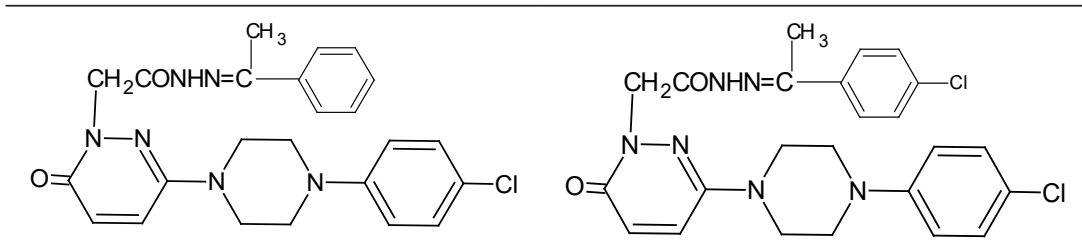

Compound 10

Compound 11 
Table 2. Elemental analysis IR and 1H NMR spectral data of 1-11 derivatives

1 Anal. Calc. For $\mathrm{C}_{23} \mathrm{H}_{23} \mathrm{ClN}_{6} \mathrm{O}_{2}: \mathrm{C}, 61.26 ; \mathrm{H}, 5.14 ; \mathrm{N}, 18.64$. Found: $\mathrm{C}, 61.38 ; \mathrm{H}, 5.21 ; \mathrm{N}, 18.83 \mathrm{IR}(\mathrm{KBr}) \mathrm{v}$ $\left(\mathrm{cm}^{-1}\right): 3185,1681,1652 .{ }^{1} \mathrm{H}$ NMR $\left(400 \mathrm{MHz}, \mathrm{DMSO}_{-}\right): \delta(\mathrm{ppm}): 11.64(\mathrm{~s}, 1 \mathrm{H}, \mathrm{NH}), 7.98$ and $8.18(1 \mathrm{H}$, $\mathrm{s}, \mathrm{s}, \mathrm{N}=\mathrm{CH}), 7.65-7.62\left(\mathrm{~d}, 1 \mathrm{H}\right.$, pyridazinone $\left.\mathrm{H}_{5}\right), 7.66-6.90(\mathrm{~m}, 9 \mathrm{H}, \mathrm{ArH}), 6.83-6.80(\mathrm{~d}, 1 \mathrm{H}$, pyridazinone $\left.\mathrm{H}_{4}\right), 5.05$ and $4.66\left(\mathrm{~s}, \mathrm{~s}, 2 \mathrm{H}, \mathrm{CH}_{2}\right), 3.38-3.36\left(\mathrm{~m}, 4 \mathrm{H}\right.$, piperazinea $\left.+\mathrm{a}^{\prime}\right), 3.28-3.26(\mathrm{~m}, 4 \mathrm{H}$, piperazineb $+\mathrm{b}$ ').

2 Anal. Calcd. For $\mathrm{C}_{23} \mathrm{H}_{23} \mathrm{ClN}_{6} \mathrm{O}_{2}: \mathrm{C}, 61.26 ; \mathrm{H}, 5.14 ; \mathrm{N}, 18.64$. Found: $\mathrm{C}, 61.46 ; \mathrm{H}, 5.10 ; \mathrm{N}, 18.43$. IR (KBr) $v$ $\left(\mathrm{cm}^{-1}\right): 3182,1683,1653 .{ }^{1} \mathrm{H}$ NMR $\left(400 \mathrm{MHz}, \mathrm{DMSO}_{6}\right): \delta(\mathrm{ppm}): 11.61(\mathrm{~s}, 1 \mathrm{H}, \mathrm{NH}), 8.18$ and $7.99(1 \mathrm{H}$, $\mathrm{s}, \mathrm{s}, \mathrm{N}=\mathrm{CH}), 7.70-6.91\left(\mathrm{~m}, 10 \mathrm{H}, \mathrm{ArH}\right.$ and pyridazinone $\left.\mathrm{H}_{5}\right), 6.86-6.78\left(\mathrm{~d}, 1 \mathrm{H}\right.$, pyridazinone $\left.\mathrm{H}_{4}\right), 5.01$ and 4.63 (s, s, $\left.2 \mathrm{H}, \mathrm{CH}_{2}\right), 3.40-3.34$ (m, 4H, piperazinea $+\mathrm{a}$ '), 3.33-3.26 (m, 4H, piperazineb+b').

3 Anal. Calc. For $\mathrm{C}_{22} \mathrm{H}_{2} \mathrm{~N}_{7} \mathrm{O}_{2}: \mathrm{C}, 63.30 ; \mathrm{H}, 5.55 ; \mathrm{N}, 23.49$. Found: $\mathrm{C}, 63.42 ; \mathrm{H}, 5.62 ; \mathrm{N}, 23.83$. IR (KBr) $v$ $\left(\mathrm{cm}^{-1}\right): 3185,1682,1658 .{ }^{1} \mathrm{H}$ NMR $\left(400 \mathrm{MHz}, \mathrm{DMSO}_{6}\right): \delta(\mathrm{ppm}): 11.60(\mathrm{~s}, 1 \mathrm{H}, \mathrm{NH}), 8.17$ and $7.98(1 \mathrm{H}$, $\mathrm{s}, \mathrm{s}, \mathrm{N}=\mathrm{CH}), 7.70-6.96\left(\mathrm{~m}, 10 \mathrm{H}, \mathrm{ArH}\right.$ and pyridazinone $\left.\mathrm{H}_{5}\right), 6.80-6.78\left(\mathrm{~d}, 1 \mathrm{H}\right.$, pyridazinone $\left.\mathrm{H}_{4}\right), 5.02$ and $4.68\left(\mathrm{~s}, \mathrm{~s}, 2 \mathrm{H}, \mathrm{CH}_{2}\right), 3.40-3.34(\mathrm{~m}, 4 \mathrm{H}$, piperazinea $+\mathrm{a}$ '), 3.33-3.25 (m, 4H, piperazineb+b').

4 Anal. Calc. for $\mathrm{C}_{24} \mathrm{H}_{25} \mathrm{ClN}_{6} \mathrm{O}_{2}$ : C, 62.00, H, 5.42, N, 18.08. Found: C, 62.13, H, 5.51, N, 18.33.IR (KBr) $v$ $\left(\mathrm{cm}^{-1}\right): 3189,1678,1661 .{ }^{1} \mathrm{H}$ NMR $\left(400 \mathrm{MHz}, \mathrm{DMSO}-\mathrm{d}_{6}\right): \delta(\mathrm{ppm}): 11.57(\mathrm{~s}, 1 \mathrm{H}, \mathrm{NH}), 7.97$ and $8.12(1 \mathrm{H}, \mathrm{s}$, $\mathrm{s}, \mathrm{N}=\mathrm{CH}), 6.97-7.63\left(\mathrm{~m}, 9 \mathrm{H}, \mathrm{ArH}\right.$ and pyridazinone $\left.\mathrm{H}_{5}\right), 6.85-6.91\left(\mathrm{~d}, 1 \mathrm{H}\right.$, pyridazinone $\left.\mathrm{H}_{4}\right), 4.60$ and 5.00 $\left(2 \mathrm{H}, \mathrm{s}, \mathrm{s}, \mathrm{CH}_{2}\right), 3.42-3.49(\mathrm{~m}, 4 \mathrm{H}$, piperazinea $+\mathrm{a})$ ') 3.18-3.35 (m, 4H, piperazineb+b'), $2.28\left(\mathrm{~s}, 3 \mathrm{H}, \mathrm{CH}_{3}\right)$.

5 Anal. Calc. for $\mathrm{C}_{24} \mathrm{H}_{25} \mathrm{ClN}_{6} \mathrm{O}_{2}: \mathrm{C}, 62.00, \mathrm{H}, 5.42, \mathrm{~N}, 18.08$. Found: $\mathrm{C}, 61.96, \mathrm{H}, 5.30, \mathrm{~N}, 18.24 . \mathrm{IR}(\mathrm{KBr}) \mathrm{v}$ $\left(\mathrm{cm}^{-1}\right): 3186,1680,1660 .{ }^{6} \mathrm{H}$ NMR $\left(400 \mathrm{MHz}, \mathrm{DMSO}-\mathrm{d}_{6}\right): \delta(\mathrm{ppm}): 11.56(1 \mathrm{H}, \mathrm{s}, \mathrm{NH}), 7.93$ and $8.13(1 \mathrm{H}, \mathrm{s}$, $\mathrm{s}, \mathrm{N}=\mathrm{CH}), 6.96-7.62\left(\mathrm{~m}, 9 \mathrm{H}, \mathrm{ArH}\right.$ and pyridazinone $\left.\mathrm{H}_{5}\right), 6.76-6.89\left(\mathrm{~d}, 1 \mathrm{H}\right.$, pyridazinone $\left.\mathrm{H}_{4}\right), 4.61$ and 5.03 $\left(2 \mathrm{H}, \mathrm{s}, \mathrm{s}, \mathrm{CH}_{2}\right), 3.41-3.47\left(\mathrm{~m}, 4 \mathrm{H}\right.$, piperazinea $+\mathrm{a}$ ') , 3.23-3.36 (m, 4H, piperazineb+b'), $2.29\left(\mathrm{~s}, 3 \mathrm{H}, \mathrm{CH}_{3}\right)$.

6 Anal. Calc. for $\mathrm{C}_{24} \mathrm{H}_{25} \mathrm{ClN}_{6} \mathrm{O}_{2}: \mathrm{C}, 62.00, \mathrm{H}, 5.42$, N, 18.08.Found: C, 61.96, H, 5.30, N, 18.24.IR (KBr) v $\left(\mathrm{cm}^{-1}\right): 3182,1677,1658 .{ }^{1} \mathrm{H}$ NMR $\left(400 \mathrm{MHz}, \mathrm{DMSO}-\mathrm{d}_{6}\right): \delta(\mathrm{ppm}): 11.58(1 \mathrm{H}, \mathrm{s}, \mathrm{NH}), 7.93$ and $8.13(1 \mathrm{H}, \mathrm{s}$, $\mathrm{s}, \mathrm{N}=\mathrm{CH}), 6.96-7.62\left(\mathrm{~m}, 9 \mathrm{H}, \mathrm{ArH}\right.$ and pyridazinone $\left.\mathrm{H}_{5}\right), 6.76-6.89\left(\mathrm{~d}, 1 \mathrm{H}\right.$, pyridazinone $\left.\mathrm{H}_{4}\right), 4.61$ and 5.03 $\left(2 \mathrm{H}, \mathrm{s}, \mathrm{s}, \mathrm{CH}_{2}\right), 3.42-3.48(\mathrm{~m}, 4 \mathrm{H}$, piperazinea $+\mathrm{a})$ ') 3.24-3.35 (m, 4H, piperazineb+b'), $2.30\left(\mathrm{~s}, 3 \mathrm{H}, \mathrm{CH}_{3}\right)$,

7 Anal. Calc. for $\mathrm{C}_{23} \mathrm{H}_{25} \mathrm{~N}_{7} \mathrm{O}_{2}: \mathrm{C}, 64.02, \mathrm{H}, 5.84, \mathrm{~N}$, 22.72.Found: C, 64.21, H, 5.60, N, 22.31.IR (KBr) v (cm $\left.{ }^{1}\right): 3181,1679,1661 .{ }^{1} \mathrm{H}$ NMR $\left(400 \mathrm{MHz}, \mathrm{DMSO}_{6}\right): \delta(\mathrm{ppm}): 11.61(1 \mathrm{H}, \mathrm{s}, \mathrm{NH}), 7.95$ and $8.14(1 \mathrm{H}, \mathrm{s}, \mathrm{s}$, $\mathrm{N}=\mathrm{CH}), 6.93-7.62\left(\mathrm{~m}, 9 \mathrm{H}, \mathrm{ArH}\right.$ and pyridazinone $\left.\mathrm{H}_{5}\right), 6.77-6.87\left(\mathrm{~d}, 1 \mathrm{H}\right.$, pyridazinone $\left.\mathrm{H}_{4}\right), 4.61$ and 5.01 $\left(2 \mathrm{H}, \mathrm{s}, \mathrm{s}, \mathrm{CH}_{2}\right), 3.41-3.47(\mathrm{~m}, 4 \mathrm{H}$, piperazinea $+\mathrm{a})$ ') 3.24-3.35 (m, 4H, piperazineb+b'), $2.30\left(\mathrm{~s}, 3 \mathrm{H}, \mathrm{CH}_{3}\right)$,

8 Anal. Calc. for $\mathrm{C}_{24} \mathrm{H}_{25} \mathrm{ClN}_{6} \mathrm{O}_{3}: \mathrm{C}, 59.94, \mathrm{H}, 5.24, \mathrm{~N}$, 17.47.Found: C, 59.63, H, 5.43, N, 17.13.IR (KBr) $v$ $\left(\mathrm{cm}^{-1}\right): 3180,1679,1659 .{ }^{1} \mathrm{H}$ NMR $\left(400 \mathrm{MHz}, \mathrm{DMSO}_{-} \mathrm{d}_{6}\right): \delta(\mathrm{ppm}): 11.50(1 \mathrm{H}, \mathrm{s}, \mathrm{NH}), 7.91$ and $8.10(1 \mathrm{H}, \mathrm{s}$, $\mathrm{s}, \mathrm{N}=\mathrm{CH}), 6.97-7.62\left(\mathrm{~m}, 9 \mathrm{H}, \mathrm{ArH}\right.$ and pyridazinone $\left.\mathrm{H}_{5}\right), 6.86-6.94\left(\mathrm{~d}, 1 \mathrm{H}\right.$, pyridazinone $\left.\mathrm{H}_{4}\right), 4.59$ and 4.99 $\left(2 \mathrm{H}, \mathrm{s}, \mathrm{s}, \mathrm{CH}_{2}\right), 3.75\left(\mathrm{~s}, 3 \mathrm{H}, \mathrm{OCH}_{3}\right), 3.36-3.43(\mathrm{~m}, 4 \mathrm{H}$, piperazinea $+\mathrm{a}$ '), 3.18-3.35 (m, 4H, piperazineb+b').

9 Anal. Calc. for $\mathrm{C}_{24} \mathrm{H}_{25} \mathrm{ClN}_{6} \mathrm{O}_{3}$ : C, 59.94, H, 5.24, N, 17.47.Found: C,59.47, H, 5.18, N, 17.24. IR (KBr) v $\left(\mathrm{cm}^{-1}\right): 3181,1680,1660 .{ }^{1} \mathrm{H}$ NMR $\left(400 \mathrm{MHz}, \mathrm{DMSO}_{6}\right): \delta(\mathrm{ppm}): 11.51(1 \mathrm{H}, \mathrm{s}, \mathrm{NH}), 7.92$ and $8.11(1 \mathrm{H}, \mathrm{s}$, $\mathrm{s}, \mathrm{N}=\mathrm{CH}), 6.95-7.63\left(\mathrm{~m}, 9 \mathrm{H}, \mathrm{ArH}\right.$ and pyridazinone $\left.\mathrm{H}_{5}\right), 6.76-6.90\left(\mathrm{~d}, 1 \mathrm{H}\right.$, pyridazinone $\left.\mathrm{H}_{4}\right), 4.60$ and 4.99 $\left(2 \mathrm{H}, \mathrm{s}, \mathrm{s}, \mathrm{CH}_{2}\right), 3.76\left(\mathrm{~s}, 3 \mathrm{H}, \mathrm{OCH}_{3}\right), 3.38-3.48(\mathrm{~m}, 4 \mathrm{H}$, piperazinea $+\mathrm{a}$ '), 3.24-3.34 (m, 4H, piperazineb+b').

10 Anal. Calc. for $\mathrm{C}_{24} \mathrm{H}_{25} \mathrm{ClN}_{6} \mathrm{O}_{2}: \mathrm{C}, 62.00 ; \mathrm{H}, 5.42 ; \mathrm{N}, 18.08$. Found: $\mathrm{C}, 62.17 ; \mathrm{H}, 5.56 ; \mathrm{N}, 17.98$. IR (KBr) $v$ $\left(\mathrm{cm}^{-1}\right): 3218,1706,1664 .{ }^{6} \mathrm{H}$ NMR (400 MHz, DMSO-d 6$): \delta(\mathrm{ppm}): 11.72(\mathrm{~s}, 1 \mathrm{H}, \mathrm{NH}), 7.73-7.70(\mathrm{~d}, 1 \mathrm{H}, \mathrm{pyr}-$ idazinone $\left.\mathrm{H}_{5}\right), 7.67-6.90\left(\mathrm{~m}, 9 \mathrm{H}\right.$, aromatic protons), 6.82-6.80 (d, $1 \mathrm{H}$, pyridazinone $\left.\mathrm{H}_{4}\right), 5.05$ and $4.70(\mathrm{~s}$, $\left.\mathrm{s}, 2 \mathrm{H}, \mathrm{CH}_{2}\right), 3.37-3.35\left(\mathrm{t}, 4 \mathrm{H}\right.$, piperazinea $+\mathrm{a}$ '), 3.27-3.25 (t, 4H, piperazineb+b'), $2.22\left(\mathrm{~s}, 3 \mathrm{H}_{1} \mathrm{CH}_{3}\right)$.

11 Anal. Calc. for $\mathrm{C}_{24} \mathrm{H}_{24} \mathrm{Cl}_{2} \mathrm{~N}_{6} \mathrm{O}_{2}: \mathrm{C}, 57.72 ; \mathrm{H}, 4.84 ; \mathrm{N}, 16.83$. Found: C, 57.96; H, 5.01; N, 16.59. IR (KBr) $v$ $\left(\mathrm{cm}^{-1}\right): 3214,1707,1666 .{ }^{1} \mathrm{H}$ NMR $\left(400 \mathrm{MHz}, \mathrm{DMSO}-\mathrm{d}_{6}\right): \delta(\mathrm{ppm}): 11.72(\mathrm{~s}, 1 \mathrm{H}, \mathrm{NH}), 7.75-7.73(\mathrm{~d}, 1 \mathrm{H}$, pyridazinone $\left.\mathrm{H}_{5}\right), 7.69-6.92\left(\mathrm{~m}, 8 \mathrm{H}\right.$, aromatic protons), 6.82-6.80 (d, $1 \mathrm{H}$, pyridazinone $\left.\mathrm{H}_{4}\right), 5.09$ and 4.72 $\left(\mathrm{s}, \mathrm{s}, 2 \mathrm{H}, \mathrm{CH}_{2}\right), 3.37-3.35\left(\mathrm{t}, 4 \mathrm{H}\right.$, piperazinea $\left.+\mathrm{a}^{\prime}\right), 3.29-3.27\left(\mathrm{t}, 4 \mathrm{H}\right.$, piperazineb+b'), $2.23\left(\mathrm{~s}, 3 \mathrm{H}, \mathrm{CH}_{3}\right)$. 
pounds 1-11 were tested against common pathogenic five Gram-positive, four Gram-negative and four Candida spp. by modified microdilution method. The antibacterial activity results of the target compounds were given in Table 3. Ampicillin trihydrate for bacteria and fluconazole for fungi were used as reference drugs. It was found that compound 3 exhibited the strongest activity against $E$. hormaechei at a MIC of $31.25 \mu \mathrm{g} / \mathrm{mL}$ and other compounds were detected moderately effective against this strain. The compounds that showed intermediate activity against $B$. subtilis subsp. subtilis and E. faecium, were found to have low activity against $S$. aureus at a MIC of $125 \mu \mathrm{g} / \mathrm{mL}$. All the tested concentrations of target compounds 1,2,3,4,8 and 10 showed high activity against $S$. maltophilia. Compounds 5 and 11 exhibited high activities against $A$. baumannii. All of the target compounds were detected to have moderate activity against $E$. faecium (VanR), K. pneumoniae, $P$. aeruginosa and $E$. coli O157:H7.
Compounds 1-11 exhibited good antifungal activity. Compound 1 showed high activity against $C$. glabrata at a MIC of $15.625 \mu \mathrm{g} / \mathrm{mL}$. Compound 9 was found less active against $C$. glabrata at a MIC of $31.25 \mu \mathrm{g} / \mathrm{mL}$. Compound 8 exhibited more significant activity against $C$. parapsilosis at a MIC of $3.9 \mu \mathrm{g} / \mathrm{mL}$ than compounds 1,5 and 9 at MICs of $7.81 \mu \mathrm{g} / \mathrm{mL}$.

Antifungal activities of compounds 1,5,6,7,8 and 9 were detected higher than the others. Especially, compounds $1,2,3,4,7,8,9,10$ and 11 showed excellent inhibition activity against $C$. tropicalis except for 5 and 6 . The MIC values of the synthesized compounds obtained against Candida spp. were given in Table 4.

When compared to ampicillin used for positive control, all of the compounds showed good activity against Gram-positive and Gram-negative strains according to statistical analysis (Scheme 2). No statistical difference was observed between compounds.

Table 3. In vitro antibacterial activity of compounds 1-11

\begin{tabular}{lccccccccc}
\hline \multicolumn{7}{c}{ Ginimal InhibitoryConcentrations $(\boldsymbol{\mu g} / \mathbf{m L})$} \\
\hline Comp. & $\mathrm{A}$ & $\mathrm{B}$ & $\mathrm{C}$ & $\mathrm{D}$ & $\mathrm{E}$ & $\mathrm{F}$ & $\mathrm{G}$ & $\mathrm{H}$ & $\mathrm{I}$ \\
\hline 1 & 125 & 125 & 125 & 62.5 & 62.5 & 125 & 125 & 125 & $*$ \\
\hline 2 & 62.5 & 125 & 125 & 62.5 & 62.5 & 125 & 125 & 62.5 & $*$ \\
\hline 3 & 125 & 125 & 125 & 31.25 & 62.5 & 125 & 125 & 62.5 & $*$ \\
\hline 4 & 125 & 62.5 & 125 & 62.5 & 62.5 & 125 & 125 & 62.5 & $*$ \\
\hline 5 & 125 & 62.5 & 125 & 62.5 & 62.5 & 31.25 & 125 & 62.5 & 31.25 \\
\hline 6 & 125 & 125 & 125 & 62.5 & 62.5 & 62.5 & 62.5 & 62.5 & 15.625 \\
\hline 7 & 62.5 & 62.5 & 125 & 62.5 & 62.5 & 62.5 & 62.5 & 62.5 & 3.9 \\
\hline 8 & 62.5 & 62.5 & 125 & 62.5 & 62.5 & 62.5 & 62.5 & 62.5 & $*$ \\
\hline 9 & 125 & 125 & 125 & 62.5 & 62.5 & 62.5 & 62.5 & 62.5 & 15.625 \\
\hline 10 & 62.5 & 62.5 & 125 & 62.5 & 62.5 & 62.5 & 62.5 & 62.5 & $*$ \\
\hline 11 & 62.5 & 125 & 125 & 62.5 & 62.5 & 31.25 & 125 & 62.5 & 15.625 \\
\hline Amp. & 125 & 31.25 & 125 & 62.5 & 7.81 & 15.625 & 7.81 & $*$ & $*$ \\
\hline A & 125 & & & $*$ & $*$ \\
\hline
\end{tabular}

A: Bacillus subtilis subsp. subtilis; B:Enterococcus faecium; C:Staphylococcus aureus; D:Enterobacterhormaechei; E: Klebsiella pneumonia; F:Acinetobacter baumannii; G: Pseudomonas aeruginosa; H: Escherichia coli; I:Stenotrophomonas maltophilia. Amp.: Ampicilline $3 \mathrm{H}_{2} \mathrm{O}$. *: No growth 
Table 4. In vitro antifungal activity of compounds 1-11

\begin{tabular}{lcccc}
\hline \multicolumn{5}{c}{ Minimal InhibitoryConcentrations $(\boldsymbol{\mu g} / \mathbf{m L})$} \\
\hline Comp. & Candida albicans & Candida glabrata & Candida parapsilosis & Candida tropicalis \\
\hline 1 & 62.5 & 15.625 & 7.81 & $*$ \\
\hline 2 & 125 & 62.5 & 15.625 & $*$ \\
\hline 3 & 62.5 & 62.5 & 15.625 & $*$ \\
\hline 4 & 125 & 62.5 & 31.25 & $*$ \\
\hline 5 & 125 & 62.5 & 7.81 & 7.81 \\
\hline 6 & 62.5 & 62.5 & 15.625 & $*$ \\
\hline 7 & 62.5 & 62.5 & 15.625 & $*$ \\
\hline 8 & 125 & 62.5 & 3.9 & $*$ \\
\hline 9 & 62.5 & 31.25 & 7.81 & $*$ \\
\hline 10 & 62.5 & 62.5 & 31.25 & $*$ \\
\hline 11 & 125 & 62.5 & 31.25 & 15.625 \\
\hline Fluconazole & $*$ & 125 & $*$ & \\
\hline
\end{tabular}

*: No growth

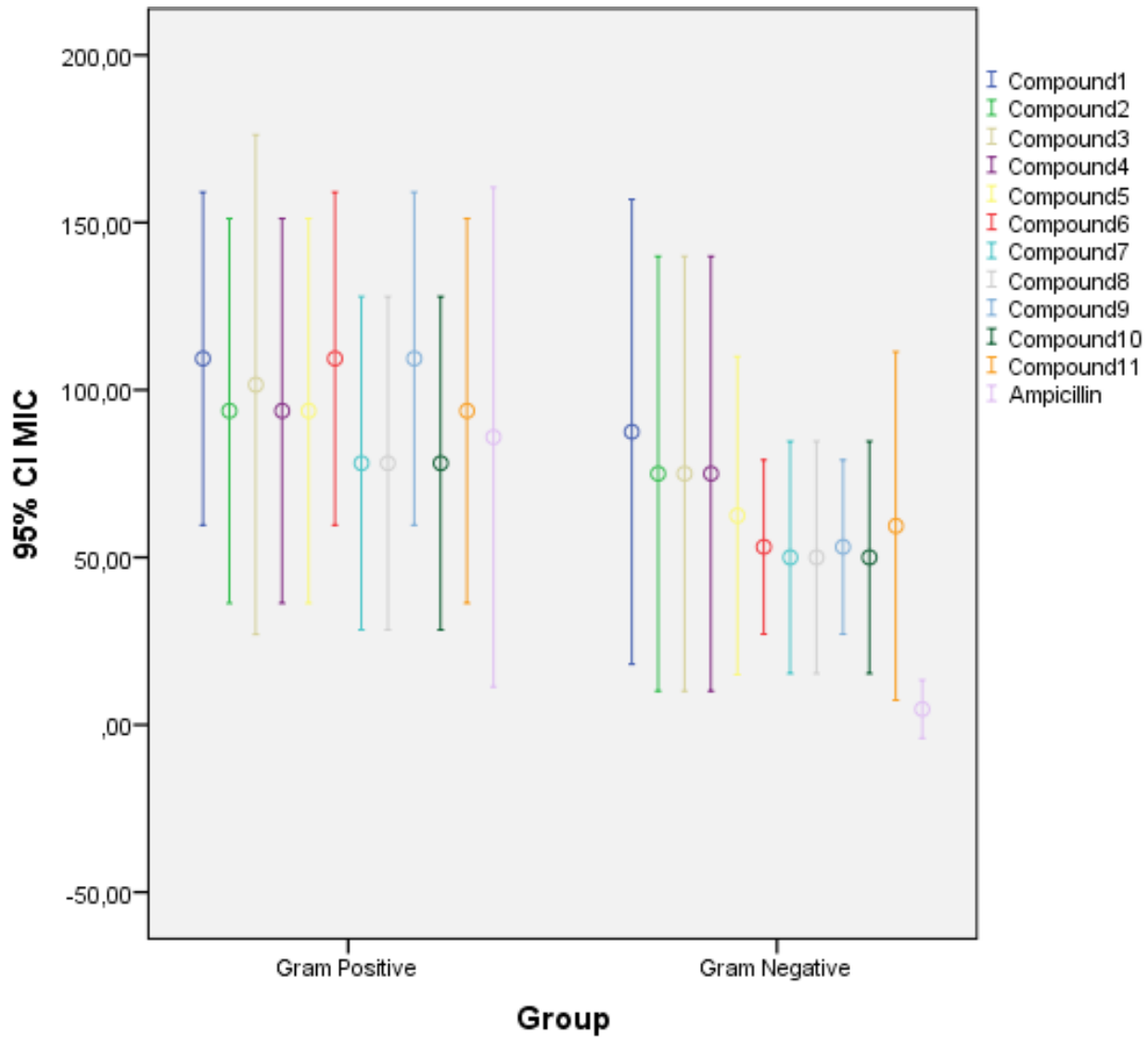

Scheme 2. Antibacterial statistical analysis of compounds 


\section{Discussions}

Due to the increasing resistance to antimicrobials, most of the antimicrobials clinically used have become ineffective in recent years (1). Therefore, many antimicrobials are thought to be inadequate in the prevention and treatment of infections. This situation could result in an increased mortality and morbidity rates of infectious diseases (2). Accordingly, the need for the treatment of multiple drug resistant (MDR) microorganisms is increasing in both community-borne and hospital-acquired infections. Centers for Disease Control and Prevention has declared that antimicrobial resistance causes failure in the treatment of MDR microorganisms such as carbapenem-resistant Enterobacteriaceae, MDR tuberculosis, MDR Acinetobacter, fluconazole-resistant Candida, vancomycin-resistant Enterococcus (VRE), MDR Pseudomonas aeruginosa and methicillin-resistant $S$. aureus (MRSA) (21).

The number of antimicrobials used in the treatment of these microorganisms are limited. $S$. aureus and coagulase-negative staphylococci are Gram-positive bacteria causing nosocomial and community acquired infections. Though MRSA infections still problem for developing countries, the rates of MRSA have declined due to the strict infection control measures taken in the West in the last decade. It is reported that MRSA rate is between $25 \%$ and $50 \%$ in blood, brain spinal fluid and other clinic samples in European Union Countries. (22).

Another life-threatening Gram-positive bacteria is $E$. faecium. It was declared that $E$. faecium is the second most frequently isolated microorganism in catheter-associated infections in the United States. High aminoglycoside and aminopenicillin resistance were reported in both E. faecalis and E. faecium isolates (23).

Gram negative-ESCAPE pathogens $(K$. pneumoniae, A. baumannii, $P$. aeruginosa, and
Enterobacter spp.) resistant to the majority of broad-spectrum antimicrobials are important etiologic agents in nosocomial infections. Karlowsky et al. conducted a study for monitoring antimicrobial resistance trends (SMART) under the global surveillance program (24). They collected samples (2113 isolate intra-abdominal and 970 isolate urinary tract infections) from 11 Latin American countries between 2013-2015. Antibiotic susceptibilities of the isolates were (2113 isolate intra-abdominal and 970 isolate urinary tract infections); amikacin 92.2\%, Enterobacter spp. $97.5 \%$, P. aeruginosa $85.3 \%$ and the sensitivity of $A$. baumannii to all tested antimicrobials was $\leq 30.9 \%$ (24). Koksal et al. reported that $E$. coli was the major pathogenic bacteria in community based urinary system infections, intensive care and intra abdominal infections in SMART study (25). Similarly, Antibiotic resistance rates of Gram-negative bacteria (E. coli, Klebsiella spp., Pseudomonas spp.) were determined to reach high levels in a meta-analysis study at West Africa (26). Studies from West Africa declared that antibiotic resistance is increasing between E. coli and Klebsiella spp. in blood and urinary system infections. Resistance to ampicillin was reported $75 \%$ and $97 \%$ in $E$. coli and Klebsiella spp. isolates, respectively (26). Furthermore, carbapenemase-producing Enterobacteriaceae are being reported 0.04$29.5 \%$ worldwide (27). The threat of infections due to carbapenemase-producing Enterobacteriaceae reveal new challenges in the treatment of inpatients and immunocompromised patients. Most of the studies emphasized that antibiotic resistance rates were higher especially in developing countries (27).

In recent years, incidence of fungal infections has increased along with anti-bacterial resistance (1,2). Fungal infections are related with AIDS, cystic fibrosis, cancers, immunocompromised or suppressed patient groups commonly. Especially, opportunistic pathogenic fungi such 
as Coccidioides immitis, Histoplasma capsulatum, Candida spp., Trichosporon spp., Cryptococcus spp., Aspergillus spp., Fusarium spp., Scedosporium spp., Pneumocystis jirovecii were detected in these patient groups (28). Among these opportunistic pathogens, Candida spp. and Aspergillus spp. are the most frequently isolated fungi from patients in intensive care units (28). Candida spp. was reported as the most seen infection agent with mortality of $38 \%$ in immunocompromised patients. C. albicans was isolated as a primary factor in $50-60 \%$ of nosocomial candidiasis. Similarly, echinocandin and azole resistance start to cause challenges in the treatment of Candida spp. in intensive care unit patients (29). It was determined that frequent use of fluconazole results in an increase of resistance in Candida spp. and C. neoformans (30). CYP5IA and CYP5IB mutations cause azole resistance in Aspergillus spp. Increase of azole resistance has been reported by many European countries such as Austria, Belgium, Denmark, Germany, France, Holland, Norway, Spain, Switzerland, UK (31).

Over the last decade, chemical structure of pyridazinones have become a striking field of study for developing new antimicrobials $(5,6)$. The synthesis of novel pyridazinone derivatives is an important point to evaluate their chemical and biological activities. In particular, the cardiovascular effects of these compounds have been studied extensively $(10,11)$. Various compounds synthesized in this area are being investigated as phosphodiesterase-III inhibitors, new antiplatelet and cardiotonic agents (8). Besides cardiovascular effects, these compounds were reported to have anti-depressant, antihypertensive, anticonvulsant, cardiotonic, antibacterial, diuretic, anti-HIV and anti-cancer effects (5-10). In particular, 3(2H)-pyridazinones are becoming remarkable therapeutic agent. Purohit et al. found that $3(2 H)$-pyridazinone derivatives were effective against $B$. megaterium, B. subtilis,
E. coli, P. fluorescens and Aspergillus species (11). Sonmez et al. detected that 5-benzoyl-4hydroxy-2-methyl-6-phenyl-2H-pyridazin-3one compound showed good inhibition activity against Gram-positive, Gram-negative bacteria and fungi isolates with MICs in the range of 0.16-0.005 $\mathrm{mg} / \mathrm{ml}$ (14). Akbas et al. reported that these compounds had the highest antimicrobial activities against Gram-positive and Gram-negative bacteria with MICs in the range of 0.31 to $<0.0024 \mathrm{mg} / \mathrm{ml}^{-1}$ (15).

Generally, in our study, antibacterial evaluation show that all compounds have moderate activity against the bacterial strains. Compound 3 shows high activity against $E$. hormaechei. All tested concentrations of compounds 1,2,3,4,8 and 10 exhibit high inhibition activity against $S$. maltophilia. All compounds show higher activity against Gram-negative bacteria and fungi than Gram-positive bacteria. In addition to this, antifungal activity of compounds 1,2,3,4,7,8,9,10 and 11 exhibit the strongest activity against $C$. tropicalis. The synthesized pyridazinone derivatives show moderate activity against $C$. albicans. It is remarkable that the antifungal activities of target compounds are high. However, benzalhydrazone derivatives (1-9) and acetophenone (10 and 11) derivatives were found to exhibit similar antimicrobial activity in this study.

\section{Conclusions}

In conclusion, our study revealed that some of the prepared 6-Substituted-3(2H)-pyridazinone-2-acetyl-2-(substituted/nonsubstitutedbenzal/acetophenone) hydrazone derivatives displayed higher MIC values than the above mentioned standard drugs. Therefore, we suggested that the compounds 1,8 and 9 might be a promising candidate of new antimicrobial agents and must be taken into consideration in the future studies. 


\section{Funding}

The study was conducted at Mersin University Advanced Technology and Research Center. This study was supported by Mersin University Scientific Research Projects Department as project number 2016-2-AP2-1908.

\section{Acknowledgment}

Statistical analysis was performed by Lecturer Sema Erden Erturk in Mersin University, Vocational School of Health Services, Mersin, Turkey.

\section{References}

1. Nolte O. Antimicrobial resistance in the 21 st century: a multifaceted challenge. Protein Pept Lett 2014; 21:3305. DOI: $10.2174 / 09298665113206660106$

2. Thabit Ak, Crandon JL, Nicolau DP. Antimicrobial resistance: impact on clinical and economic outcomes and the need for new antimicrobials. Expert Opin Pharmacother 2015;16(2):159-77. DOI: $10.1517 / 14656566.2015 .993381$

3. MacCarthy MW, Kontoyiannis DP, Cornely OA, Perfect JR, Walsh TJ. Novel Agents and Drug Targets to Meet the Challenges of Resistant Fungi. J Infect Dis 2017;216:474-83. DOI: 10.1093/infdis/jix130

4. Rai M, Ingle AP, Pandit R, Paralikar P, Gupta I, Chaud MV, Dos Santos CA. Broadenin the spectrum of small-molecule antibacterials by metallic nanoparticles to overcome microbial resistance. Int J Pharm 2017;532:139-148. DOI: 10.1016/j.ijpharm.2017.08.127

5. Ibrahim HM, Behbehani H, Elnagdi MH. Approaches towards the synthesis of a novel class of 2-amino-5-arylzonicotinate, pyridazinone and pyrido[2,3-d] pyrimidine derivatives as potent antimicrobial agents. Chem Cent J 2013;7(1):123. DOI: 10.1186/1752153X-7-123

6. Singh J, Sharma D, Bansal R. Pyridazinone:an attractive lead for ant-inflamatory and analgesic drug discovery. Future Med Chem 2017;9(1):95-127. DOI: 10.4155/fmc-2016-0194

7. Jaballah MY, Serya RT, Abouzid K. Pyridazinone Based Scaffods as Privileed Structures in anti-cancer Therapy. Drug Res (Stuttg) 2017;67(3):138-48. DOI: 10.1055/s-0042-119992

8. Asif M. A mini review on biological activities pf pyridazinone derivatives as antiulcer, antisecretory, antihistamin and particularly against histamine H3R. Mini
Rev Med Chem 2015;14(3):1093-103. DOI: 10.2174/1 389557514666141127143133

9. Sukuroglu M, Onkol T, Onurdag FK, Akalin G, Sahin MF. Synthesis and in vitro biological activity of new 4,6-disubstituted 3(H)-pyridazinone-acetohydrazide derivatives. Z Naturforsch C 2012;67(5-6):257-65. DOI: $10.5560 /$ ZNC.2012.67c0257

10. Bansal R, Sridhar T. Pyridazin-3(2H)-ones: the versatile pharmacophore of medicinal significance. Med Chem Res 2013;22:2539-52. DOI: 10.1007/s00044012-0261-1

11. Purohit DM, Shah VH. Novel method for synthesis and antimicrobial evaluation of 2-aroyl-6-hydroxy/chloro/ hydrazino/carboxymethoxy-3(2H)-pyridazinones. Heterocycl Commun 1997;3 (3):267-272. DOI: 10.1515/ HC.1997.3.3.267

12. Islam M, Siddiqui AA, Rajesh R. Synthesis antitubercular, antifungal and antibacterial activities of 6-susbtituted phenyl-2-(3'-substituded phenyl pyridazin-6'-yl)-2,3,4,5-tetrahydropyridazin-3-one. Acta Pol Pharm 2008;65(3):353-62.

13. Ozcelik AB, Gokce M, Orhan I, Kaynak F, Sahin MF. Synthesis and antimicrobial, acetylcholinesterase and butyrycholinesterase inhibitory activities of novel ester and hydrazide derivatives of $3(\mathrm{H})$-pyridazinone. Arnzeimittelforschung 2010;60(7):452-8.

14. Sonmej M, Berber L, Akbas B. Synthesis, antibacterial and antifungal activity of some new pyridazinone metal complexes. Eur J Med Chem 2005;41:101-5. DOI: 10.1016/j.ejmech.2005.10.003

15. Akbas E, Berber I. Antibacterial and antifungal activities of new pyrazolo $[3,4-d]$ pyridazin derivatives, Eur J Med Chem 2005;40:401-5. DOI: 10.1016/j.ejmech.2004.12.001

16. Utku S, Gokce M, Aslan G, Bayram G, Ulger M, Emekdaş $\mathrm{G}$ et al. Synthesis and in vitro antimycobacterial activities of novel 6-substituted-3(2H)-pyridazinone-2-acetyl-2-(substituted/nonsubstituted acetophenone) hydrazine. Turk J Chem 2011;35(2):331-9.

17. Gokce M, Utku S, Kupeli E. Synthesis and analgesic and anti-inflammatory activities 6-substituted-3(2H)-pyridazinone-2-acetyl-2-(p-substituted/nonsubstituted benzal) hydrazone derivatives. Eur J Med Chem 2009;44:3760-64. DOI: 10.1016/j.ejmech.2009.04.048

18. Jorgensen JH, Ferraro MJ. Antimicrobial suspectibility testing: general principles and comtemporary. Clin Infect Dis 1998;26:973-80. DOI: 10.1086/513938

19. Woods GL, Washington JA, Antibacterial susceptibility tests: dilution and disk diffusion methods. In: Murray PR, Baron EJ, Pfaller MA, Tenover FC, Yolken RH, eds. Manual of clinical microbiology. 6th ed. Washington, DC: American Society for Microbiology; 1995:1327-41.

20. Clinical Laboratory Standards Institute. Performance Standards For Antimicrobial Susceptibility Testing; 
Twenty-Second Informational Supplement. CLSI. M02-A11 and M07-A9, 2012:40-61.

21. https://www.cdc.gov/drugresistance/threat-report-2013/pdf/ar-threats-2013-508.pdf. [Last accested 1 August 2017].

22. Lob SH, Badal RE, Hackel MA, Sahm DF. Epidemiology and antimicrobial Suspectibility of Gram-Negative Pathogens Causing Intra-abdominal Infections in Pediatrics Patients in Europe-SMART 2011-2014. J Pediatric Infect Dis Soc 2017;6(1):72-79

23. Akova M. Epidemiology of antimicrobial resistance in bloodstream infections. Virulence 2016;7(3):252-66. DOI: $10.1080 / 21505594.2016 .1159366$

24. Karlowksy JA, Hoban DJ, Hackel MA, Lob SH, Sahm DF. Resistance among Gram-negative ESKAPE pathogens isolated from hospitalized patients with intra-abdominal and urinary tract infections in Latin American countries: SMART 2013-2015. Braz J Infect Dis 2017;21(3):343-8. DOI: 10.1016/j.bjid.2017.03.006

25. Koksal F, Yilmaz G, Unal S, Zarakolu P, Korten V, Mulazzimoglu L et al. Epidemiology and susceptibility of pathogens from SMART 2011-12 Turkey: evaluation of hospital-acquired versus community-acquired urinary tract infections and ICU- versus non-ICU-associated intra-abdominal infections. J Antimicrob Chemo- ther 2017;72: 1364-72. DOI: 10.1093/jac/dkw574

26. Bernabe KJ, Langendorf C, Ford N, Ronat JB, Murphy RA. Antibiotic resistance in West Africa: a systematic review and meta-analysis. International Journal of Antimicrobial Agent 2017;17: 30274-1.

27. Kelly AM, Mathema B, Larson EL. Carbapenem-resistant Enterobacteriaceae in the community: a scoping review. Int J Antimicrob Agents 2017;50:127-134. DOI: 10.1016/j.ijantimicag.2017.03.012

28. Srinivasan A, Lopez-Ribot JL, Ramasubramanian AK. Overcoming antifungal resistance. Drug Discov Today Technol 2014:65-71. DOI: 10.1016/j.ddtec.2014.02.005

29. Maubon D, Garnaud C, Calandra T, Sanglard D, Cornet M. Resistance of Candida spp. to antifungal drugs in the ICU: where are we now?. Intensive Care Med 2014;40:1241-55. DOI: 10.1007/s00134-014-3404-7

30. Ben-Ami R, Olshtain-Pops K, Krieger M et al. Antibiotic exposure as a risk factor for fluconazole-resistant Candida bloodstream infection. Antimicrob Agents Chemother 2012; 56: 2518-2523. DOI: 10.1128/ AAC.05947-11

31. Arendrup MC. Update on antifungal resistance in Aspergillus and Candida Clin Microbiol Infect 2014; 20 (Suppl. 6): 42-48. 